\title{
Real Time Ultrasound Needle Image Simulation Using Multi-dimensional Interpolation
}

\author{
Mengchen Zhu ${ }^{1}$ and Septimiu E. Salcudean ${ }^{2}$ \\ 1 Department of Biomedical Engineering, Georgia Institute of Technology, \\ Atlanta, GA, USA \\ mczhu@gatech.edu \\ 2 Department of Electrical and Computer Engineering, \\ University of British Columbia, Vancouver, BC, Canada \\ tims@ece.ubc.ca
}

\begin{abstract}
In this paper, we propose an interpolation-based method for simulating needle images in B-mode ultrasound. We parametrize the needle image as a function of needle position and orientation. We collect needle images under various spatial configurations in a water-tank using a guidance robot. Then we use multi-dimensional tensor-product interpolation to simulate images of needles with arbitrary poses and positions using the collected images. Interpolated needle images are superimposed on top of phantom image backgrounds. The similarity between the simulated and the real images is measured using a correlation metric. A comparison with in-vivo images is also performed. The simulation procedure is demonstrated using transverse needle images and extended to sagittal needle images and brachytherapy seed images. The proposed method could be used in clinical procedure training simulators.
\end{abstract}

\section{Introduction}

The simulation of needle insertion under ultrasound guidance would be useful in the planning and training of a number of clinical procedures. A needle insertion simulator would include a haptic interface to enable interaction with a deformable tissue model and a real time ultrasound image simulator to provide visual feedback. The image simulator requires both tissue and needle images to be rendered. Previous work (e.g. 7 713 ) addressed primarily the simulation of B-mode images of soft tissue; the needle was simply rendered as a bright line in the longitudinal plane, without details or validation.

Needle images are highly view-dependent, with images changing drastically with the relative location between the needle and the ultrasound transducer. Physicians exploit this dynamic effect to judge the position of the needle, especially in the transverse plane [2]. For example, in prostate brachytherapy [5], physicians insert the needle until they see its tip, then rotate it to insure that the tip is at the desired transverse position. For simulation purposes, it is important to take into account the view-dependency property of needles.

In this paper, we propose to use interpolation on collected needle sample images to simulate new needle images in real time. The collected images are

T. Jiang et al. (Eds.): MICCAI 2010, Part II, LNCS 6362, pp. 429 436, 2010.

(C) Springer-Verlag Berlin Heidelberg 2010 
of various needle poses, so the simulator is able to reproduce view-dependent features of the needle images.

The classical way of simulating ultrasound images by interpolation is by slicing a 3D volume [8] and applying techniques such as voxel nearest-neighbor [9]. This approach cannot deal with view-dependent features of needle images. Indeed, looking at the needle from another angle is not equivalent to rotating the original image. Approaches based on physical acoustic models 44 are computationally expensive, while the faster methods using artificial acoustic models [7]3] lack accuracy. Hybrid methods [1] can be view-dependent and real-time, but they only simulate point scatterers which are not suitable models of needles, which have much more complex forms of scattering, such as the "comet tail" artifact. The idea of using images of an object under various poses and positions to simulate new object poses has been explored in the field of image-based rendering (e.g. [6]), but the ray-based parametrization from such references cannot be adopted to our case because there is no relative motion between the "camera" and the "light source" in our case - both are the ultrasound transducer.

\section{Simulation Methods}

It is noted that the image of the needle in water resembles that in the phantom/tissue. The basic idea of the simulation is therefore to first simulate the needle images in water by interpolating sample images collected in water and then superimpose the simulated images onto the tissue background.

We make the assumption that the needle image depends only on the local spatial configuration (position and orientation) of the intersection of the needle with the imaging plane. In this manner, we can represent the planar needle image as a function $I_{n}: \mathbb{R}^{6} \rightarrow \mathbb{R}^{2}$ of the six degrees of freedom of the needle.

In the following, we first demonstrate our approach of parametrization, sample collection, interpolation, and validation on the transverse plane of a transrectal ultrasound (TRUS) probe. Using a slightly different parametrization, the same method could be applied to simulate images on the sagittal (longitudinal) plane and seed images.

\subsection{Parametrization}

The parametrization for the needle configuration with respect to the TRUS probe transverse plane is illustrated in Fig. 1a Assuming the ultrasound beam profile has a constant thickness with respect to $r$ along the $Z$ (elevation) direction and the needle is inserted toward the negative $Z$ direction, we define the imaging plane as the plane through which the needle enters the beam profile. This plane can be determined experimentally by inserting the needle until the tip starts to show up. We attach a Cartesian frame $\underline{C}_{p}$ at the probe origin $\underline{\sim}_{p}$ in the imaging plane and another Cartesian frame $\underline{C}_{n}$ at the intersection of the imaging plane and the needle axis $\underset{\sim}{P}$. $Z_{n}$ coincides with the needle axis and $Y_{n}$ indicates the bevel tip direction. We use $(r, \theta)$ to denote the polar coordinates of ${\underset{\sim}{P}}_{n}$ 


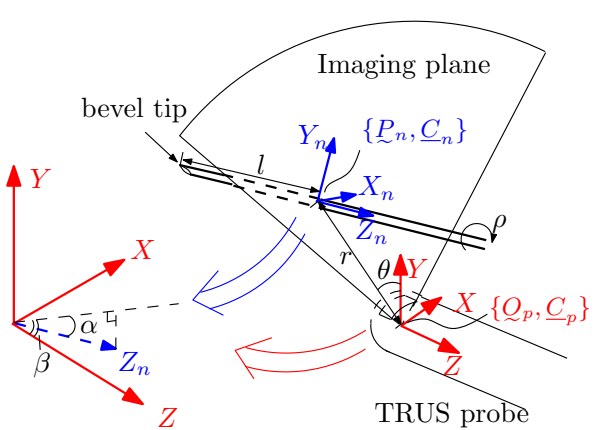

(a)

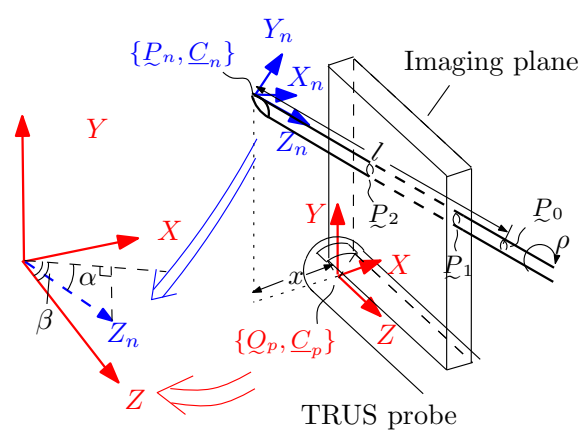

(b)

Fig. 1. Coordinate assignments for: (a) Transverse (b) Sagittal plane

in $\left\{Q_{p}, \underline{C}_{p}\right\}$, and $l$ to denote the needle depth. We describe the rotation transformation between $\underline{C}_{p}$ and $\underline{C}_{n}$ using roll-pitch-yaw angles $(\rho, \alpha, \beta)$. The needle image is assumed to be a function $I_{n}(r, \theta, l, \rho, \alpha, \beta)$ of these spatial variables. We have shown experimentally that the needle image is invariant with respect to $\theta$. Indeed, if we keep other spatial parameters constant and vary $\theta$, the image will only rotate by $\theta$ but will not change in intensity or shape otherwise:

$$
I_{n}(r, \theta, l, \rho, \alpha, \beta)=R_{\theta}\left(I_{n}\left(r, 0, l, \rho_{0}, \alpha_{0}, \beta_{0}\right)\right)
$$

where $R_{\theta}$ is a $2 \mathrm{D}$ rotation transform of the image with respect to the ultrasound probe center and $(\rho, \alpha, \beta)$ and $\left(\rho_{0}, \alpha_{0}, \beta_{0}\right)$ are related by the same rotation transform. This rotation invariance is due to the curvilinear geometry of the TRUS probe. To obtain images at an arbitrary $\theta$, we rotate the image at $\theta=0$. Thus the function to be sampled and interpolated is $I_{n}\left(r, 0, l, \rho_{0}, \alpha_{0}, \beta_{0}\right): \mathbb{R}^{5} \rightarrow \mathbb{R}^{2}$.

\subsection{Tensor-Product Interpolation}

Assuming that sample values of $I_{n}$ are available through a collection method as described in the next section, the interpolation scheme seeks to estimate values of $I_{n}$ at non-sample points. The speed of interpolation is of major concern. Nearest neighbor is the fastest interpolation method available, and is twice as fast as linear interpolation 12. To strike a balance between speed and accuracy, we look for a scheme that is a hybrid of the nearest neighbor and linear interpolation. A suitable method is the tensor-product interpolation which enables the use of different piecewise polynomial interpolations - nearest neighbor and linear interpolations being special cases - along different dimensions [1]. The choice between the nearest neighbor and the linear interpolation for each dimension is based on the fact that during the needle insertion, the needle position on the imaging plane and its orientation are constrained by the tissue, therefore not likely to change much. It is the depth and roll angle that are under the physician's control and therefore responsible for the continuous changes of the 


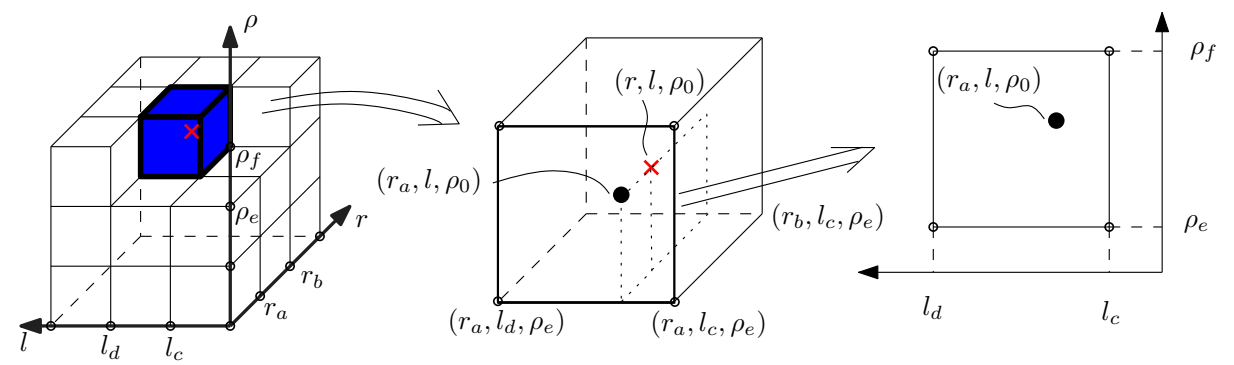

Fig. 2. Visualization of the interpolation process. Here only 3 dimensions are drawn. Each vertex represents a sample image. The red cross represents the queried configuration $\left(r, l, \rho_{0}\right)$.

needle images. Using linear interpolation for $l, \rho$ enables us to simulate these changes, and using nearest neighbor for other dimensions speeds up the interpolation considerably. Furthermore, using nearest neighbor for $r$ will eliminate artifacts caused by averaging two images with features at different locations (e.g. the bright tail).

A general account of the tensor-product interpolation scheme is available in [1. A key feature of the method is that changing the sequence of interpolation does not change the result, therefore we could perform nearest neighbor interpolation first to reduce the computational cost. Specifically we use nearest neighbor interpolation for $r, \alpha, \beta$ and linear interpolation for $l, \rho$. An operational procedure of the scheme applied to our problem is as follows and the key steps are visualized in Fig. 2,

1. Given the queried coordinate $(r, l, \rho, \alpha, \beta)$, calculate the rotated coordinate $\left(r, l, \rho_{0}, \alpha_{0}, \beta_{0}\right)$ in Eq. 1 ,

2. Find the enclosing hypercube in the 5 -dimensional sample space that contains the rotated coordinate: $\left(r, l, \rho_{0}, \alpha_{0}, \beta_{0}\right) \in\left[r_{a}, r_{b}\right] \times\left[l_{c}, l_{d}\right] \times\left[\rho_{e}, \rho_{f}\right] \times$ $\left[\alpha_{g}, \alpha_{h}\right] \times\left[\beta_{i}, \beta_{j}\right]$.

3. Find the nearest neighbors for $r, \alpha_{0}, \beta_{0}$; say $r_{a}, \alpha_{h}, \beta_{j}$ respectively;

4. In the rectangular subspace $\left[l_{c}, l_{d}\right] \times\left.\left[\rho_{e}, \rho_{f}\right]\right|_{\left(r_{a}, \alpha_{h}, \beta_{j}\right)} \in \mathbb{R}^{2}$, use linear interpolation to get the interpolant image $\tilde{I}_{n}$. This is just bi-linearly interpolating $I_{n}\left(r_{a}, l_{c}, \rho_{e}, \alpha_{h}, \beta_{j}\right), I_{n}\left(r_{a}, l_{c}, \rho_{f}, \alpha_{h}, \beta_{j}\right), I_{n}\left(r_{a}, l_{d}, \rho_{e}, \alpha_{h}, \beta_{j}\right)$, and $I_{n}\left(r_{a}, l_{d}, \rho_{f}, \alpha_{h}, \beta_{j}\right)$;

5. Now $\tilde{I}_{n}$ corresponds to a simulated needle image with the needle at coordinates $\left(r_{a}, 0\right)$ in the imaging plane. To simulate the image at $(r, \theta)$, we first translate $\tilde{I}_{n}$ by $\left(r_{a}-r\right)$ and then rotate by $\theta$ according to Eq. 1 to finally get the approximated $I_{n}(r, \theta, l, \rho, \alpha, \beta)$.

It can be shown that steps 1-4 of the above algorithm have a computational cost of $\mathcal{O}\left(N_{p}\right)$, where $N_{p}$ is the number of pixels of the images being interpolated. In the current implementation $N_{p}=80 \times 350$ and is the size of needle images cropped from the original $640 \times 480$ images (the rest is black background). The 
speed of step 5 above largely depends on the interpolation method used for the spatial image rotations. In Matlab, using the nearest-neighbor will result in real time performance but may introduce aliasing artifacts or "jaggies". This was eliminated in an OpenGL implementation where linear interpolation can be computed in real time. On a PC with an Intel $2.2 \mathrm{GHz}$ dual core CPU, the overall algorithm can achieve an average frame rate of 44 FPS for a resolution of $640 \times 480$ in the Matlab implementation without major optimization efforts.

\subsection{Sample Collection and Superimposition}

All data collection was done in a water tank of size $\mathrm{L} \times \mathrm{W} \times \mathrm{H}=50 \times 30 \times$ $20 \mathrm{~cm}^{3}$ filled with degassed water at $20^{\circ} \mathrm{C}$. Brachytherapy needles (Bard ${ }^{\circledR}, 18$ gauge) were imaged with the TRUS probe of an ultrasound machine (Ultrasonix SonixRP). The machine parameters were set to be the same as in a brachytherapy procedure, except for disabled Time Gain Control, single focus, and sound speed $c=c_{\text {water }}$. The time gain control was disabled during the data collection because in water there is very little attenuation with increasing depth; single focus was used because multiple focal points may sometimes cause split images in water. The samples were collected in the water tank rather than in a phantom because in water moving from one needle configuration to another leaves no needle tracks. Furthermore, the needle images in water are much more clearly defined.

A needle guidance robot previously developed [10] was used for the data collection. Since the robot does not move in $l$ or $\rho$, we controlled these two variables by hand. The robot was programmed to remotely connect to the SonixRP and capture images automatically after each desired configuration was reached.

The range and sample numbers of each dimension are shown in the caption of Fig. 3. The ranges of $\alpha, \beta$ were kept small, as in a real brachytherapy procedure. Only positive rotation angles were collected due to symmetry.

The accuracy of the tensor-product interpolation depends on the spatial sampling rate. To ensure that the sampling rate is high enough, test images were first captured on a fine sample grid, then part of these images were used to interpolate the rest. Correlation was then used to compare the results, and the interpolated images were found to be similar to the real images when the sample numbers in Fig. 3 were used. As an example, for the roll angle $\rho$, when using images collected at every $180^{\circ}$ to interpolate images of $45^{\circ} \rho$ intervals, the correlation between the interpolated and the real images was 0.93 ; when using images collected at every $90^{\circ}$, the correlation was 0.97 .

To simulate the needle images in the tissue, background images of a gelatine tissue mimicking phantom were collected, onto which the interpolated needle images in water were superimposed by addition. Superimposition by taking the maximum of the foreground and the background has also been tested, but the results looked less realistic. It was concluded that superimposition by addition gives satisfactory results. Note that the success of addition relies on the approximate linearity of the imaging process, which may be influenced by machine settings such as the time-gain-compensation. 


\section{Results and Validation}

To validate the simulation method, 43 needle images in both the water tank and a homogeneous gelatine phantom with cellulose scatterers were collected using the same machine settings as used when the needle image samples were collected. The needle configurations were chosen to be different from those previously collected. Blank phantom background images were also collected before each insertion for use in the superimposition. Because images of the same modality are being compared, classical normalized cross-correlation is used as the similarity metric.

To begin with, we only measure the similarities between the areas adjacent to the needle in the images. This is justified because it is the needle image that is localized most of the time during the brachytherapy procedure and because the

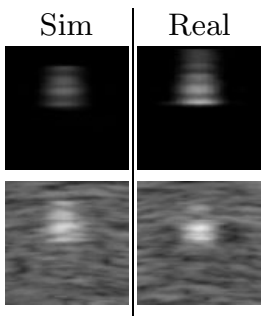

(a) $(6.5,7.5,0,2,0)$

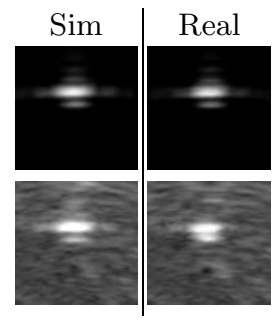

(b) $(4.8,6,0,0,0)$

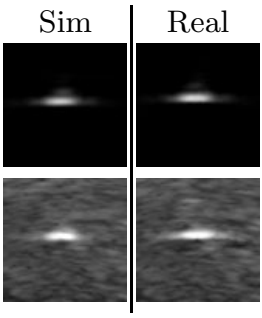

(c) $(4.5,6,135,0,0)$

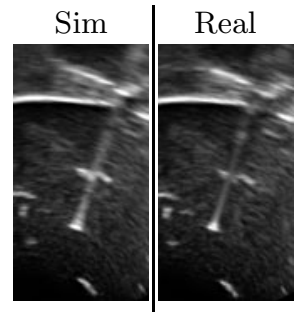

(d) Bright tail

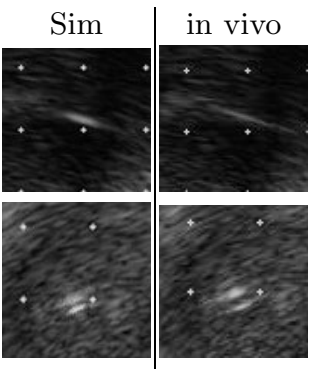

(e) Transverse in vivo

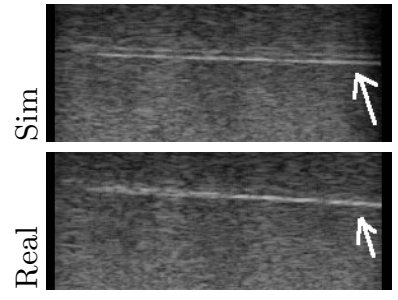

(f) Sagittal phantom

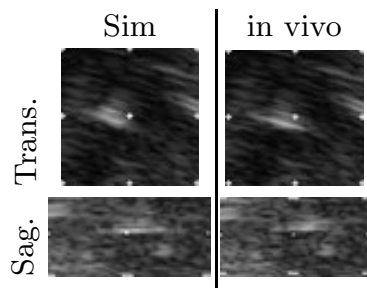

(g) Seeds in vivo

Fig. 3. (a) through (c): comparison of simulated and real needle images of the transverse plane. upper-left quadrant: simulated image in water; upper-right: real image in water; lower-left: simulated image in phantom; lower-right: real image in phantom. Configurations are of the form $(r, l, \rho, \alpha, \beta)$. The range and sample number of variables are: $r: 30 \sim 65 \mathrm{~mm} ; 8$ samples; $l: 1 \sim 22 \mathrm{~mm} ; 12$ samples; $\rho: 0 \sim 180^{\circ} ; 3$ samples; $\alpha: 0 \sim 9^{\circ} ; 4$ samples; $\beta$ : $0 \sim 15^{\circ} ; 3$ samples; (d) Simulated and phantom needle image with bright tails; (e) Comparison of transverse simulated and in vivo needle images from a brachytherapy session; (f) Comparison of simulated and phantom sagittal needle images. The range and sample number of variables are: $x:-0.7 \sim$ $1.5 \mathrm{~cm} ; 23$ samples; $\rho: 0 \sim 180^{\circ} ; 3$ samples; $\alpha: 0 \sim 6^{\circ} ; 3$ samples $\beta: 0 \sim 15^{\circ} ; 4$ samples; (g) Comparison of the simulated and in vivo seed images in the transverse (upper) and sagittal plane (lower). 
background contributes to the false positive measure of the correlation score. For each of the 43 image pairs, an $80 \times 80$ region around the the needle image was cropped out and correlations were calculated. The mean correlation between the simulated and the real needle images in water is $\operatorname{cor}_{w}=0.86$ out of 1 . The mean correlation between the simulated (after superimposition) and the real needle images in phantom is $\operatorname{cor}_{p}=0.79$.

In some occasions, a bright tail is visible distal to the needle image due to reverberation inside the needle. Our interpolation and superimposition method produces images similar to real images when the tail is present. The correlation between regions that contain the tail region and the phantom images has a typical score of 0.89 , as shown in Fig. $3 \mathrm{~d}$.

The simulation results were also compared with several in vivo needle images collected during a brachytherapy session. To obtain a similar background for superimposition, the needles in the in vivo images were first erased by copying surrounding background using the image editing software GIMP (GNU Image Manipulation Program). Then the simulated needle images were superimposed. Example results are shown in Fig. 3e. Since there were no direct measurements of the needle position and orientation in-vivo, the simulated images were generated with the assumptions that the needle tip was in the imaging plane and that the needle was parallel to the $Z$ axis (0 pitch and yaw angles). It is evident from Fig. 3e that under these conditions the simulated needle images are similar to the in vivo ones. The difference may be attributed to the different ultrasound machines being used for data collection - an Ultrasonix ${ }^{\circledR}$ machine was used for the sample image collection; a BK Medical ${ }^{\circledR}$ machine was used in the hospital.

Using slightly different parametrization and collection methods, the same method could be extended to simulate needle insertion in the sagittal plane and radioactive seeds in both planes in real-time (Fig. 1b sagittal plane coordinate assignment; Fig. 3f] and $3 \mathrm{~g}$ results)).

\section{Discussion and Future Work}

This paper presents a first view-dependent real-time needle image simulation method that is also suitable for simulating other wire-like objects such as radioactive seeds. The use of a robot to collect pose-dependent sample images proves to be accurate, efficient, and repeatable. The simulated images are not only visually appealing, but also accurate. The accuracy was validated objectively using a correlation metric and by comparison with in vivo data. The simulation method is compatible with existing real-time deformable tissue image simulations. Indeed, the needle simulation described here has recently been successfully incorporated into a real-time prostate brachytherapy simulator [3].It should be noted that the range of positions that our method can handle is only limited by the physical movement range of the brachytherapy robot used for data collection. Given more samples, the method can readily deal with a larger parameter range with little increased computational cost.

The simulation may potentially serve as a real-time "front-end" for a physically based simulator where sample images are simulated rather than collected. 
Furthermore, the collected image sample database may potentially be used as training database for seed detection purposes.

In this paper, it was assumed that the needle does not change its pitch and yaw angles during the insertion. However, if the needle is not rigid, the pitch and yaw angles might be continuously changing when the needle is pushed sideways. Linear interpolation for these angles and piecewise rigid approximations may be needed to better simulate a bending needle.

\section{References}

1. Allen, M., Isaacson, E.: Numerical Analysis for Applied Science. Wiley Interscience, Hoboken (1998)

2. Chapman, G., Johnson, D., Bodenham, A.: Visualisation of needle position using ultrasonography. Anaesthesia 61(2), 148 (2006)

3. Goksel, O., Salcudean, S.: Haptic simulator for prostate brachytherapy with simulated ultrasound. In: Bello, F., Cotin, S. (eds.) Biomedical Simulation. LNCS, vol. 5958, pp. 150-159. Springer, Heidelberg (2010)

4. Jensen, J., Nikolov, I.: Fast simulation of ultrasound images. In: Ultrasonics Symposium, vol. 2. IEEE, Los Alamitos (2000)

5. Langley, S., Laing, R.: Prostate brachytherapy has come of age: a review of the technique and results. BJU International 89(3), 241-249 (2002)

6. Levoy, M., Hanrahan, P.: Light field rendering. In: Proceedings of the 23rd annual conference on Computer graphics and interactive techniques, pp. 31-42. ACM, New York (1996)

7. Magee, D., Zhu, Y., Ratnalingam, R., Gardner, P., Kessel, D.: An augmented reality simulator for ultrasound guided needle placement training. Medical and Biological Engineering and Computing 45(10), 957-967 (2007)

8. Maul, H., Scharf, A., Baier, P., Wuestemann, M., Guenter, H., Gebauer, G., Sohn, C.: Ultrasound simulators: experience with the SonoTrainer and comparative review of other training systems. Ultrasound in Obstetrics and Gynecology 24(5), 581-585 (2004)

9. Rohling, R., Gee, A., Berman, L.: A comparison of freehand three-dimensional ultrasound reconstruction techniques. Med. Image Anal. 3(4), 339-359 (1999)

10. Salcudean, S., Prananta, T., Morris, W., Spadinger, I.: A robotic needle guide for prostate brachytherapy. In: IEEE International Conference on Robotics and Automation, ICRA 2008, pp. 2975-2981 (2008)

11. Shams, R., Hartley, R., Navab, N.: Real-time simulation of medical ultrasound from CT images. In: Metaxas, D., Axel, L., Fichtinger, G., Székely, G. (eds.) MICCAI 2008, Part II. LNCS, vol. 5242, pp. 734-741. Springer, Heidelberg (2008)

12. Thevenaz, P., Blu, T., Unser, M.: Image interpolation and resampling. In: Handbook of Medical Imaging, Processing and Analysis, pp. 393-420 (2000)

13. Vidal, F., John, N., Healey, A., Gould, D.: Simulation of ultrasound guided needle puncture using patient specific data with 3D textures and volume haptics. Computer Animation and Virtual Worlds 19(2), 111 (2008) 